\title{
Evaluation of aflatoxin contamination of soybean in Rwanda,
}

\author{
M. Niyibituronsa ${ }^{1,2,3}$, A.N. Onyango ${ }^{2}$, S. Gaidashova ${ }^{1}$, S. Imathiu ${ }^{2}$, M. Uwizerwa ${ }^{1}$, I. Wanjuki ${ }^{3}$, \\ F. Munga ${ }^{3}$, S. Mutiga ${ }^{3}$ and J. Harvey ${ }^{3}$ \\ ${ }^{1}$ Rwanda Agriculture Board, Rwanda, ${ }^{2}$ Jomo Kenyatta University of Agriculture and Technology and ${ }^{3}$ Biosciences \\ eastern and central Africa-International Livestock Research Institute Hub, Kenya
}

Mycotoxin contamination is a global threat to food and feed safety ${ }^{(1)}$. Soybean is one of the food commodities which are susceptible to colonization by mycotoxin-producing moulds, including those that produce aflatoxin and trichothecenes ${ }^{(2)}$. Aflatoxin, produced by different Aspergillus species, have been shown to contribute to, among other health problems, liver cancer and immunosuppressant, and is correlated with stunting of growth and development in children ${ }^{(3)}$. High levels of aflatoxin contamination have been reported in some commodities across the East African region ${ }^{(4)}$. However, there is limited or no information about the extent of mycotoxin contamination in soybean. We therefore aimed to determine the extent of aflatoxin contamination in soybean, and to assess people's awareness about mycotoxins in Rwanda.

A survey was conducted at farms within three agro ecological zones suitable for soybean production in 2015. Soybean samples were collected from farmers $(n=300)$ who also completed questionnaires about pre-and post-harvest farm practices, and aflatoxin awareness. The concentration of aflatoxin (ppb) in individual soybean samples was tested by enzyme-linked immunosorbent assay (ELISA) using a commercially-available kit, according to manufacturer instructions ${ }^{(5)}$.

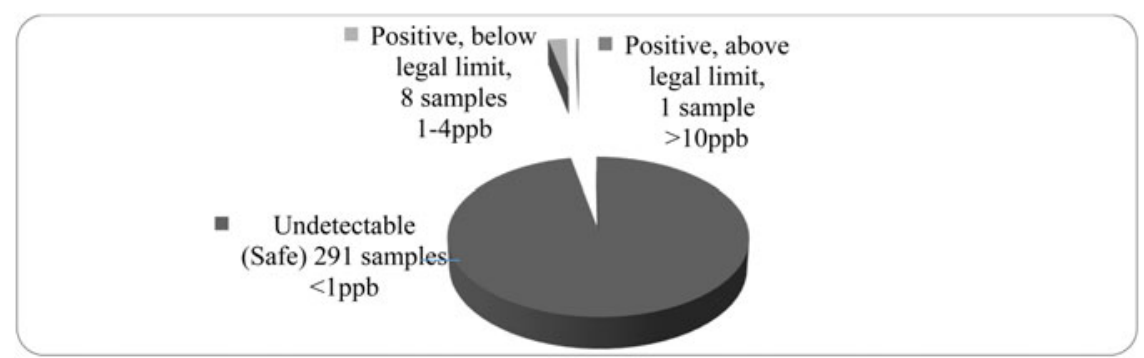

Fig. 1

The majority of the samples had aflatoxin below the regulatory limits of 4, 10, $20 \mathrm{ppb}$ set by the European Union (EU), the World Food Programme/Kenya, and the US Food and Drug Administration, respectively. Only one sample had a concentration above the most stringent EU limit. The awareness survey showed that $7.3 \%$ of the respondents were aware of aflatoxin contamination. This study shows that soybean is safe for human consumption. Given its importance as a major protein source in diet, there is need to promote the cultivation and consumption of Soybean in Rwanda. There is need to create awareness about mycotoxins among the Rwandese consumers of cereal crops that are vulnerable to colonization by mycotoxigenic fungi.

This project was supported by the BecA-ILRI Hub through the Africa Biosciences Challenge Fund (ABCF) program.

1. Wild C.P \& Gong Y.Y, (2010) Oxford J, Carcinogenesis; 31(1): 71-82.

2. Nesheim S \& Wood G.E, (1995) AOCS J, 72, no.12.

3. European Food Safety Authority, (2013) EN-406.

4. Kaaya A.N (2005) CPP R8435 (ZA0653)\& R8442 (ZA0666).

5. Helica Biosystems, (2007) Cat.No. 981AFL01LM. 